\title{
Next-to-Leading-Order QCD Corrections to Higgs Boson Plus Jet Production with Full Top-Quark Mass Dependence
}

\author{
S. P. Jones, M. Kerner, and G. Luisoni \\ Max-Planck-Institut für Physik, Föhringer Ring 6, 80805 München, Germany
}

(Received 1 February 2018; published 16 April 2018)

\begin{abstract}
We present the next-to-leading-order QCD corrections to the production of a Higgs boson in association with one jet at the LHC including the full top-quark mass dependence. The mass of the bottom quark is neglected. The two-loop integrals appearing in the virtual contribution are calculated numerically using the method of sector decomposition. We study the Higgs boson transverse momentum distribution, focusing on the high $p_{t, H}$ region, where the top-quark loop is resolved. We find that the next-to-leading-order QCD corrections are large but that the ratio of the next-to-leading-order to leading-order result is similar to that obtained by computing in the limit of large top-quark mass.
\end{abstract}

DOI: 10.1103/PhysRevLett.120.162001

Introduction.-During Run I and early Run II of the LHC great progress has been made in establishing many of the properties of the Higgs boson particle discovered in 2012. Already the spin and $C P$ properties are well constrained and its couplings to the standard model (SM) weak vector bosons and heavier fermions (top quarks and tau leptons) have been measured [1]. So far the measured properties are consistent with the predictions of the SM.

Among the different channels for the production of a SM Higgs boson, gluon fusion, which we consider here, is the mechanism yielding the largest contribution. At lowest order in perturbation theory this process is mediated by a closed loop of heavy quarks and next-to-leading-order (NLO) QCD corrections therefore require the computation of two-loop contributions. A Higgs effective field theory (HEFT) was derived long ago [2], in which the heavy quarks are integrated out and the Higgs boson couples directly to the gluons. This allows the computations to be simplified considerably.

One interesting regime to consider is that of Higgs boson production with a transverse momentum $p_{t, H}$ of the order of the top-quark mass, $m_{T}$, or larger. Here the top-quark loop is resolved and it becomes possible to disentangle the SM contribution from effects of new physics. However, in this regime finite top-quark mass effects are not negligible and the effective theory approximation becomes increasingly poor. In other words, events in which the Higgs boson is recoiling against one or more jets acquiring a large transverse momentum do not fall into the validity range of the effective field theory description. It is thus important to

Published by the American Physical Society under the terms of the Creative Commons Attribution 4.0 International license. Further distribution of this work must maintain attribution to the author(s) and the published article's title, journal citation, and DOI. Funded by SCOAP . go beyond the HEFT approximation and include finite topquark mass effects to obtain reliable predictions in this kinematical range.

Within the HEFT approximation corrections to inclusive Higgs boson production are known to $\mathrm{N}^{3} \mathrm{LO} Q \mathrm{CD}$ accuracy [3], while the fully differential corrections for $H+1$ jet production are known to next-to-next-to-leading-order (NNLO) QCD accuracy [4-7]. Finite quark mass corrections to $H+1$ jet production have been known at $\mathrm{LO}$ for a long time [8,9] and LO results are also known for the higher multiplicity processes $H+2$ jets $[10,11]$ and recently $H+$ 3 jets $[12,13]$. The HEFT results for $H+1$ jet production have also been supplemented by an expansion in $1 / m_{T}^{2}$ at NLO QCD accuracy $[14,15]$ and combined with the exact Born and real corrections [16]. They were also included in multipurpose Monte Carlo generators to produce merged samples matched to parton showers [17-19] and used to improve the Higgs NNLO QCD transverse momentum distributions in the HEFT above the top-quark mass threshold [20].

One of the first major steps towards the computation of the full two-loop NLO QCD virtual corrections was made in Ref. [21], where the planar master integrals were computed analytically in the Euclidean region and shown to contain elliptic integrals. At the same time an expansion valid in the limit of small bottom-quark mass allowed insight to be gained into the NLO QCD effects due to nearly massless quarks [22-25]. Very recently a NLO QCD result expanded in the regime where the Higgs boson and top-quark masses are small, relevant for the description of the Higgs boson transverse momentum distribution at large $p_{t, H} \gtrsim 400 \mathrm{GeV}$, was also studied [26,27].

On the experimental side, recently the CMS collaboration has considered events where the Higgs boson transverse momentum $p_{t, H}$ is larger than $450 \mathrm{GeV}$ [28], a feat made possible through the use of boosted techniques [29], 
which allow the Higgs boson to be identified through its decay to bottom quarks.

In this Letter we present the first NLO QCD computation of Higgs boson production in association with one jet retaining the full top-quark mass dependence. In the following sections we present the computational setup used for this calculation and selected phenomenological results.

Computational setup.-We compute using conventional dimensional regularization with $d=4-2 \epsilon$. The top-quark mass is renormalized in the on-shell scheme and the QCD coupling and gluon wave function in the $\overline{\mathrm{MS}}$ scheme with $n_{f}=5$ light quarks, with the top-quark loops subtracted at zero momentum. The top-quark mass renormalization is performed by inserting the mass counterterm into the heavy quark propagators. Alternatively, the mass renormalization can be calculated by taking the derivative of the one-loop amplitude with respect to $m_{T}$. We have used both methods as a cross-check.

The sampling of the phase-space generator has been adapted to generate a nearly uniform distribution of points in $p_{t, H}$.

Born and real radiation.-The computation of everything but the virtual amplitudes is performed within the POWHEG-BOX-V2 framework [30], taking advantage of the existing HJ generator [31] for HEFT, in which the Born and real radiation amplitudes are computed using MADGRAPH4 $[32,33]$ and the virtual amplitudes are taken from MCFM [34]. The subtraction of the infrared divergences is performed using FKS [35].

We supplemented this code with the analytical Born amplitudes with full top-quark mass dependence from Ref. [9], whereas the one-loop real radiation contribution was generated with GoSAM $[36,37]$ using the BLHA $[38,39]$ interface developed in Ref. [40]. For the purpose of this computation GoSAM has been improved such that it now automatically switches to quadruple precision in regions where the amplitude becomes unstable due to one of the final state partons becoming soft or collinear to another parton. The amplitudes generated by GoSAM are computed at run time with NINJA [41-43] using the quadninja feature. The scalar one-loop integrals are computed with the ONELOOP [44] integral library. As a consistency check the virtual amplitudes in HEFT were cross-checked with GoSAM, whereas the real radiation amplitudes in the full theory were compared against OPENLOOPS [45].

The virtual amplitude.-The Lorentz structure of the $H \rightarrow q \bar{q} g$ and $H \rightarrow g g g$ partonic amplitudes can be decomposed, after imposing parity conservation, transversality of the gluon polarization vectors, and the Ward identity, in terms of 2 and 4 tensor structures, respectively; see for example Ref. [46]. This decomposition is not unique. For the $H \rightarrow g g g$ amplitude we follow Ref. [47] and choose to decompose it such that three of the form factors (which multiply the tensor structures) are related by cyclic permutations of the external gluon momenta while the fourth is itself invariant under such permutations. For the $H \rightarrow q \bar{q} g$ amplitude our decomposition is chosen such that the form factors are related by interchanging the external quark and antiquark momenta. We separately compute all form factors and use these symmetries as a cross-check of our result.

In order to compute the amplitudes we closely follow the method of Refs. [48,49]. We construct projection operators for each of the form factors and contract them with the amplitude omitting external spinors and polarization vectors. This procedure allows us to write the amplitude in terms of scalar integrals.

The Feynman diagrams contributing to the two-loop virtual amplitude are generated using QGRAF [50] and further processed using ReduZE [51], GinAC [52], FERMat [53], and MATHEMATICA. We cross-checked the amplitudes with expressions obtained from a two-loop extension of GoSAM, which uses QGRAF and FORM [54,55]. The integrals appearing in the amplitude are reduced to master integrals using a customized version of the program REDUZE. To simplify the numerical evaluation we choose a quasifinite basis of master integrals [56]. The resulting integrals are calculated numerically using SECDEC [57,58]. For the numerical integration we use a quasi-Monte Carlo method based on a rank-one lattice rule [59-61]. Neglecting crossings we evaluate a total of 102 planar and 18 nonplanar two-loop integrals.

The Higgs boson mass is set to $m_{H}=125 \mathrm{GeV}$ and the top-quark mass is chosen such that $m_{H}^{2} / m_{T}^{2}=12 / 23$, which means that $m_{T}=173.055 \mathrm{GeV}$. Fixing the ratio of the Higgs boson to top-quark mass allows us to reduce by 1 the number of independent scales appearing in the two-loop virtual amplitudes. This simplifies the integral reduction and the form of the resulting reduced amplitude.

We subtract the infrared and collinear poles of the virtual amplitude to obtain the finite part of the virtual amplitude $\mathcal{V}_{\text {fin }}$ as required in the POWHEG-BOX-V2 framework [62]. The IR subtraction procedure requires the one-loop amplitudes up to order $\epsilon^{2}$, which we compute numerically using the same procedure as for the two-loop amplitudes.

Phenomenology.-In this section we present results for $H+1$ jet production at the LHC at a center-of-mass energy of $13 \mathrm{TeV}$. Jets were clustered using the anti-kt jet algorithm implemented in FASTJET [63-65] with a radial distance of $R=0.4$ and requiring a minimum transverse momentum of $p_{t, j}>30 \mathrm{GeV}$. We used the PDF4LHC15_nlo_30_pdfas [66-69] set interfaced through LHAPDF [70] for both LO and NLO predictions, and fixed the default value of factorization and renormalization scales $\mu_{F}$ and $\mu_{R}$ to $H_{T} / 2$, defined as

$$
\frac{H_{T}}{2}=\frac{1}{2}\left(\sqrt{m_{H}^{2}+p_{t, H}^{2}}+\sum_{i}\left|p_{t, i}\right|\right),
$$

where the sum runs over all final state partons $i$. This scale is known to give a good convergence of the perturbative expansion and stable differential $K$ factors (ratio of NLO to LO predictions) in the effective theory [71]. To estimate the theoretical uncertainty we vary independently $\mu_{F}$ and $\mu_{R}$ by 
TABLE I. Total cross sections at LO and NLO in the HEFT and $\mathrm{FT}_{\text {approx }}$ approximations and with full top-quark mass dependence. The upper and lower values due to scale variation are also shown. More details can be found in the text.

\begin{tabular}{lcc}
\hline \hline Theory & LO $[\mathrm{pb}]$ & NLO $[\mathrm{pb}]$ \\
\hline HEFT: & $\sigma_{\mathrm{LO}}=8.22_{-2.15}^{+3.17}$ & $\sigma_{\mathrm{NLO}}=14.63_{-2.54}^{+3.30}$ \\
FT $_{\text {approx }}:$ & $\sigma_{\mathrm{LO}}=8.57_{-2.24}^{+3.31}$ & $\sigma_{\mathrm{NLO}}=15.07_{-2.54}^{+2.54}$ \\
Full: & $\sigma_{\mathrm{LO}}=8.57_{-2.24}^{+3.31}$ & $\sigma_{\mathrm{NLO}}=16.01_{-3.73}^{+1.59}$ \\
\hline \hline
\end{tabular}

factors of 0.5 and 2 , and exclude the opposite variations. The total uncertainty is taken to be the envelope of this seven-point variation.

To better highlight the differences arising from the twoloop massive contributions, we compare the new results with full top-quark mass dependence, which we label as "full theory result" or simply "full" in the following, to two different approximations. In addition to predictions in the effective theory, which are referred to as HEFT in the following, we show results in which everything but the virtual amplitudes is computed with full top-quark mass dependence. In this latter case only the virtual contribution is computed in the effective field theory and reweighted by the full theory Born amplitude for each phase-space point. Following Ref. [72] we call this prediction "approximated full theory" and label it as $\mathrm{FT}_{\text {approx }}$ from now on.

We start by presenting the total cross sections, which are reported in Table I. For comparison we present results also for the HEFT and $\mathrm{FT}_{\text {approx }}$ approximations.

Together with the prediction obtained with the central scale defined according to Eq. (1) we show the upper and lower values obtained by varying the scales. While at LO the top-quark mass effects lead to an increase of $4.3 \%$, at NLO this increase is of the order of $9 \%$ compared to the HEFT approximation, and there is an increase of about $6 \%$ in the total NLO cross section when comparing the $\mathrm{FT}_{\text {approx }}$ result with the full theory one. It is important to keep in mind that when taking into account massive bottom-quark loop contributions, the interference effects are sizable and cancel to a large extent the increase in the total cross section observed here between the HEFT and the full theory results (see, e.g., the results in Ref. [13]). Note, however, that the bottom-quark mass effects at $\mathrm{LO}$ are of the order of $2 \%$ or smaller above the top-quark threshold.

Considering more differential observables, it is well known that very significant effects due to resolving the top-quark loop are displayed by the Higgs boson transverse momentum distribution, which is softened for larger values of $p_{t, H}$ by the full top-quark mass dependence. By considering the high energy limit of a pointlike gluon-gluon Higgs interaction and one mediated via a quark loop it is possible to derive the scaling of the squared transverse momentum distribution $d \sigma / d p_{t, H}^{2}[73,74]$, which drops as $\left(p_{t, H}^{2}\right)^{-1}$ in the effective theory, and goes instead as $\left(p_{t, H}^{2}\right)^{-2}$ in the full

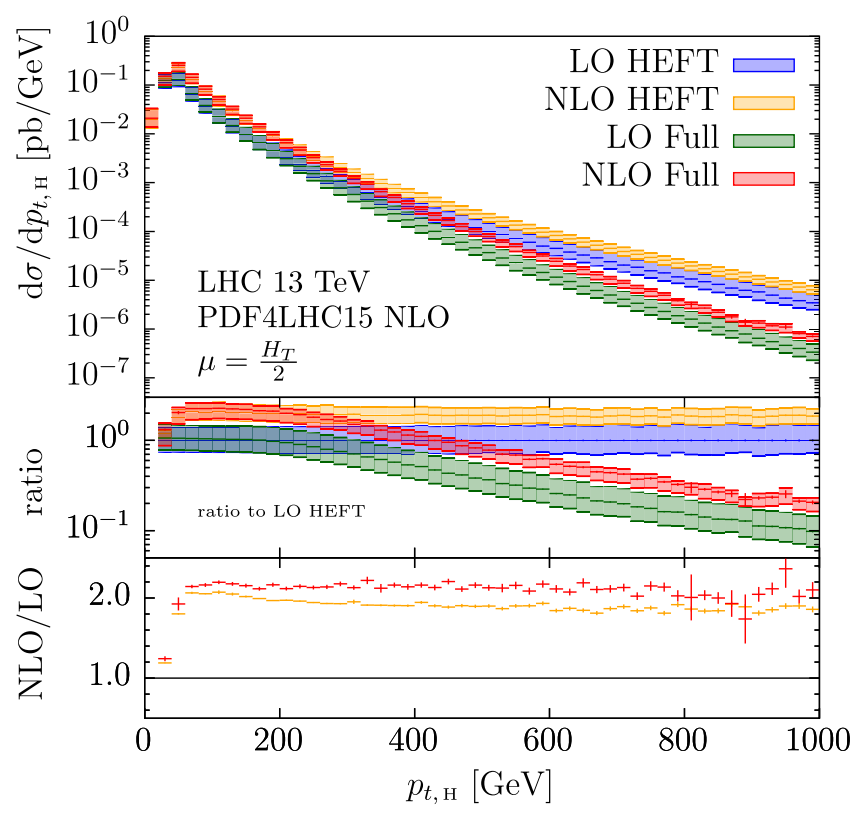

FIG. 1. Higgs boson transverse momentum spectrum at LO and NLO in QCD in HEFT and with full top-quark mass dependence. The upper panel shows the differential cross sections; in the middle panel we normalize all distributions to the LO HEFT prediction and in the lower panel we show the differential $K$ factors for both the HEFT and the full theory distributions. More details can be found in the text.

theory. This fact was shown to hold numerically at LO for up to three jets in Ref. [13]. It is interesting to verify this also after NLO QCD corrections are applied. To do so, in Fig. 1 we show the transverse momentum spectrum of the Higgs boson at LO and NLO in the HEFT approximation and with the full top-quark mass dependence.

In the upper panel we display each differential distribution with the theory uncertainty band originating from scale variation. To highlight the different scaling in $p_{t, H}$, in the middle panel we normalize all the distributions to the LO curve in the effective theory. It is thus possible to see that for low transverse momenta the full theory predictions overshoot slightly the effective theory ones. For $p_{t, H}>200 \mathrm{GeV}$ the two predictions start deviating more substantially. At LO the two uncertainty bands do not overlap any more above $400 \mathrm{GeV}$, whereas at NLO this happens already around $340 \mathrm{GeV}$ due to reduction of the uncertainty at this order. The logarithmic scale also allows us to see that the relative scaling behavior within the two theory descriptions is preserved between LO and NLO. The curves in the lowest panel of Fig. 1 show the differential $K$ factor in HEFT and in the full theory. In both cases above $150 \mathrm{GeV}$ they become very stable and amount to about 1.95 and 2.2, respectively. Thus the NLO corrections are large also in the full theory. This broadly agrees with the conclusions of Ref. [27], where the expanded result showed a similar enhancement of the $K$ factor by about $6 \%$ in the tail compared to the HEFT. 


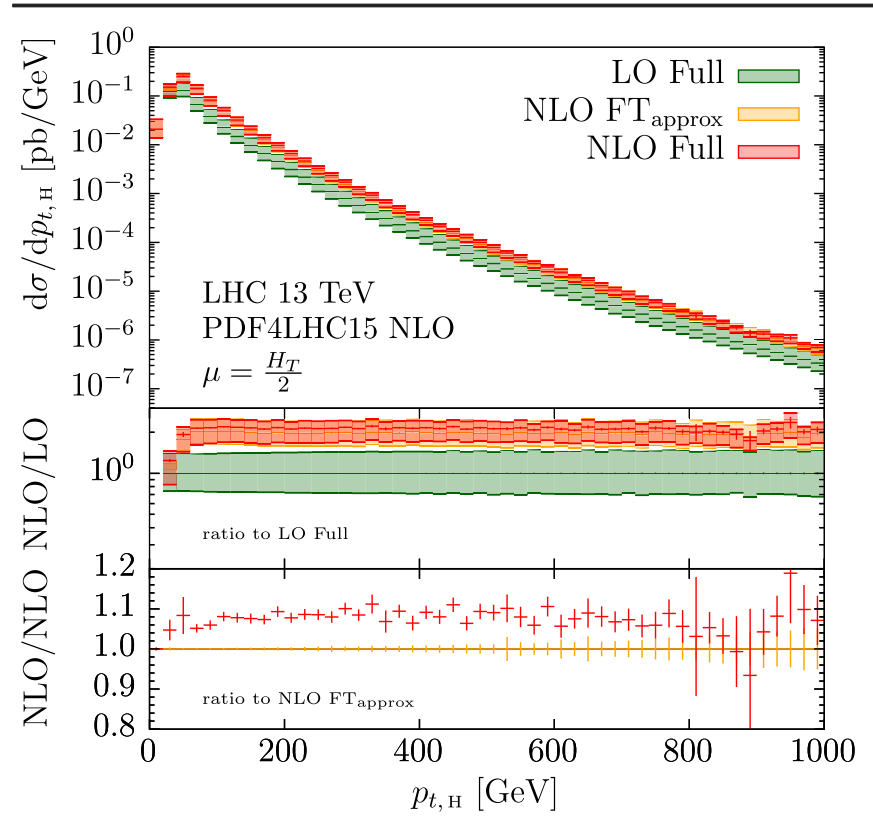

FIG. 2. Higgs boson transverse momentum spectrum at LO and NLO in QCD with full top-quark mass dependence compared with the NLO predictions in $\mathrm{FT}_{\text {approx }}$. The upper panel shows the differential cross sections; in the middle panel we normalize all distributions to the LO prediction and in the lower panel we show the differential ratio between the NLO $\mathrm{FT}_{\text {approx }}$ predictions and the full theory ones. More details can be found in the text.

To conclude this section we compare the new predictions for the Higgs boson transverse momentum with the one in $\mathrm{FT}_{\text {approx }}$. At $\mathrm{LO}$ the two predictions are identical by construction; it is, however, interesting to check how good $\mathrm{FT}_{\text {approx }}$ can reproduce the full theory results. In the main panel of Fig. 2 we plot the three curves. To highlight better the differences among the two predictions, in the middle panel we normalize the distributions to the LO prediction. This allows us to compare the two differential $K$ factors, which behave very similarly over the full kinematical range. As already observed in the case of double Higgs boson production [49], the scale uncertainty band of the full theory predictions is slightly reduced compared to the one in $\mathrm{FT}_{\text {approx }}$. In order to quantify the difference between the two predictions, in the lower panel we display the ratio of the full NLO curve to the $\mathrm{FT}_{\text {approx }}$ NLO curve. We observe that the full top-quark mass virtual contribution enhances the predictions obtained by reweighting the HEFT virtual by an almost constant factor of about $8 \%$.

Conclusions and outlook.-In this Letter we have presented for the first time NLO QCD corrections to Higgs boson plus jet production retaining the full top-quark mass dependence. We observe that the size of the NLO corrections is large but, for our choice of the renormalization and factorization scale, the $K$ factor is approximately constant above the top-quark threshold. Compared to $\mathrm{FT}_{\text {approx }}$ predictions, the full two-loop contribution enhances the NLO predictions by about $6 \%$ at the level of the total cross section and by about $8 \%$ at the level of the differential transverse momentum distribution for $p_{t, H} \gtrsim 200 \mathrm{GeV}$. Despite a completely different $p_{t}$ scaling, the $K$ factors in the HEFT and in the full theory behave in a very similar way above $200 \mathrm{GeV}$.

The result removes the theoretical uncertainty on differential $H+1$ jet distributions due to the unknown top mass corrections at NLO in QCD. Besides the transverse momentum distribution, shown here, this calculation enables accurate predictions to be made also for other observables where the top-quark mass effects may play a significant role.

As the experimental precision at the LHC improves in the coming years, this result aids the study of the Higgs boson properties also in boosted regimes. Providing a more accurate theoretical description of the Higgs boson production at large transverse momentum will be helpful not only for unraveling the details of the electroweak symmetry breaking mechanism, but also in the search for indirect signs of new physics.

We thank Gudrun Heinrich for encouraging us to undertake this computation and also for numerous discussions and comments on the manuscript. We thank Nigel Glover and Hjalte Frellesvig for interesting discussions and for their insightful comments on the Lorentz structure of the amplitude. We thank Tiziano Peraro for helping us in the upgrade of the NINJA interface. We thank Sophia Borowka for interesting discussions. This research was supported by the Munich Institute for Astro- and Particle Physics (MIAPP) of the DFG cluster of excellence "Origin and Structure of the Universe." S.P. J. was supported by the Research Executive Agency (REA) of the European Union under Grant No. PITN-GA2012316704 (HiggsTools) during part of this work. We gratefully acknowledge support and resources provided by the Max Planck Computing and Data Facility (MPCDF).

[1] G. Aad et al. (ATLAS and CMS Collaborations), J. High Energy Phys. 08 (2016) 045.

[2] F. Wilczek, Phys. Rev. Lett. 39, 1304 (1977).

[3] C. Anastasiou, C. Duhr, F. Dulat, E. Furlan, T. Gehrmann, F. Herzog, A. Lazopoulos, and B. Mistlberger, J. High Energy Phys. 05 (2016) 058.

[4] R. Boughezal, F. Caola, K. Melnikov, F. Petriello, and M. Schulze, J. High Energy Phys. 06 (2013) 072.

[5] X. Chen, T. Gehrmann, E. W. N. Glover, and M. Jaquier, Phys. Lett. B 740, 147 (2015).

[6] R. Boughezal, F. Caola, K. Melnikov, F. Petriello, and M. Schulze, Phys. Rev. Lett. 115, 082003 (2015).

[7] R. Boughezal, C. Focke, W. Giele, X. Liu, and F. Petriello, Phys. Lett. B 748, 5 (2015).

[8] R. K. Ellis, I. Hinchliffe, M. Soldate, and J. J. van der Bij, Nucl. Phys. B297, 221 (1988).

[9] U. Baur and E. W. N. Glover, Nucl. Phys. B339, 38 (1990).

[10] V. Del Duca, W. Kilgore, C. Oleari, C. Schmidt, and D. Zeppenfeld, Phys. Rev. Lett. 87, 122001 (2001).

[11] V. Del Duca, W. Kilgore, C. Oleari, C. Schmidt, and D. Zeppenfeld, Nucl. Phys. B616, 367 (2001). 
[12] F. Campanario and M. Kubocz, Phys. Rev. D 88, 054021 (2013).

[13] N. Greiner, S. Höche, G. Luisoni, M. Schönherr, and J.-C. Winter, J. High Energy Phys. 01 (2017) 091.

[14] R. V. Harlander, T. Neumann, K. J. Ozeren, and M. Wiesemann, J. High Energy Phys. 08 (2012) 139.

[15] T. Neumann and M. Wiesemann, J. High Energy Phys. 11 (2014) 150.

[16] T. Neumann and C. Williams, Phys. Rev. D 95, 014004 (2017).

[17] M. Buschmann, D. Goncalves, S. Kuttimalai, M. Schonherr, F. Krauss, and T. Plehn, J. High Energy Phys. 02 (2015) 038.

[18] K. Hamilton, P. Nason, and G. Zanderighi, J. High Energy Phys. 05 (2015) 140.

[19] R. Frederix, S. Frixione, E. Vryonidou, and M. Wiesemann, J. High Energy Phys. 08 (2016) 006.

[20] X. Chen, J. Cruz-Martinez, T. Gehrmann, E. W. N. Glover, and M. Jaquier, J. High Energy Phys. 10 (2016) 066.

[21] R. Bonciani, V. Del Duca, H. Frellesvig, J. M. Henn, F. Moriello, and V. A. Smirnov, J. High Energy Phys. 12 (2016) 096.

[22] R. Mueller and D. G. Öztürk, J. High Energy Phys. 08 (2016) 055.

[23] K. Melnikov, L. Tancredi, and C. Wever, J. High Energy Phys. 11 (2016) 104.

[24] K. Melnikov, L. Tancredi, and C. Wever, Phys. Rev. D 95, 054012 (2017).

[25] J. M. Lindert, K. Melnikov, L. Tancredi, and C. Wever, Phys. Rev. Lett. 118, 252002 (2017).

[26] K. Kudashkin, K. Melnikov, and C. Wever, J. High Energy Phys. 02 (2018) 135.

[27] J. M. Lindert, K. Kudashkin, K. Melnikov, and C. Wever, arXiv:1801.08226.

[28] A. M. Sirunyan et al. (CMS Collaboration), Phys. Rev. Lett. 120, 071802 (2018).

[29] J. M. Butterworth, A. R. Davison, M. Rubin, and G. P. Salam, Phys. Rev. Lett. 100, 242001 (2008).

[30] S. Alioli, P. Nason, C. Oleari, and E. Re, J. High Energy Phys. 06 (2010) 043.

[31] J. M. Campbell, R. K. Ellis, R. Frederix, P. Nason, C. Oleari, and C. Williams, J. High Energy Phys. 07 (2012) 092.

[32] T. Stelzer and W. F. Long, Comput. Phys. Commun. 81, 357 (1994).

[33] J. Alwall, P. Demin, S. de Visscher, R. Frederix, M. Herquet, F. Maltoni, T. Plehn, D. L. Rainwater, and T. Stelzer, J. High Energy Phys. 09 (2007) 028.

[34] J. M. Campbell, R. K. Ellis, and C. Williams, Phys. Rev. D 81, 074023 (2010).

[35] S. Frixione, Z. Kunszt, and A. Signer, Nucl. Phys. B467, 399 (1996).

[36] G. Cullen, N. Greiner, G. Heinrich, G. Luisoni, P. Mastrolia, G. Ossola, T. Reiter, and F. Tramontano, Eur. Phys. J. C 72, 1889 (2012).

[37] G. Cullen et al., Eur. Phys. J. C 74, 3001 (2014).

[38] T. Binoth et al., Comput. Phys. Commun. 181, 1612 (2010).

[39] S. Alioli et al., Comput. Phys. Commun. 185, 560 (2014).

[40] G. Luisoni, P. Nason, C. Oleari, and F. Tramontano, J. High Energy Phys. 10 (2013) 083.

[41] P. Mastrolia, E. Mirabella, and T. Peraro, J. High Energy Phys. 06 (2012) 095; 11 (2012) 128(E).
[42] H. van Deurzen, G. Luisoni, P. Mastrolia, E. Mirabella, G. Ossola, and T. Peraro, J. High Energy Phys. 03 (2014) 115.

[43] T. Peraro, Comput. Phys. Commun. 185, 2771 (2014).

[44] A. van Hameren, Comput. Phys. Commun. 182, 2427 (2011).

[45] F. Cascioli, P. Maierhofer, and S. Pozzorini, Phys. Rev. Lett. 108, 111601 (2012).

[46] T. Gehrmann, M. Jaquier, E. W. N. Glover, and A. Koukoutsakis, J. High Energy Phys. 02 (2012) 056.

[47] M. Boggia et al., arXiv:1711.09875.

[48] S. Borowka, N. Greiner, G. Heinrich, S. P. Jones, M. Kerner, J. Schlenk, U. Schubert, and T. Zirke, Phys. Rev. Lett. 117, 012001 (2016); 117, 079901(E) (2016).

[49] S. Borowka, N. Greiner, G. Heinrich, S. P. Jones, M. Kerner, J. Schlenk, and T. Zirke, J. High Energy Phys. 10 (2016) 107.

[50] P. Nogueira, J. Comput. Phys. 105, 279 (1993).

[51] A. von Manteuffel and C. Studerus, arXiv:1201.4330.

[52] C. W. Bauer, A. Frink, and R. Kreckel, J. Symb. Comput. 33, 1 (2002).

[53] R. Lewis, Computer algebra system fermat, http://home .bway.net/lewis/.

[54] J. Vermaseren, arXiv:0010025.

[55] J. Kuipers, T. Ueda, J. Vermaseren, and J. Vollinga, Comput. Phys. Commun. 184, 1453 (2013).

[56] A. von Manteuffel, E. Panzer, and R. M. Schabinger, J. High Energy Phys. 02 (2015) 120.

[57] S. Borowka, G. Heinrich, S. P. Jones, M. Kerner, J. Schlenk, and T. Zirke, Comput. Phys. Commun. 196, 470 (2015).

[58] S. Borowka, G. Heinrich, S. Jahn, S. P. Jones, M. Kerner, J. Schlenk, and T. Zirke, Comput. Phys. Commun. 222, 313 (2018).

[59] Z. Li, J. Wang, Q.-S. Yan, and X. Zhao, Chin. Phys. C 40, 033103 (2016).

[60] J. Dick, F. Y. Kuo, and I. H. Sloan, Acta Numer. 22, 133 (2013).

[61] D. Nuyens and R. Cools, Math. Comput. 75, 903 (2006).

[62] G. Heinrich, S. P. Jones, M. Kerner, G. Luisoni, and E. Vryonidou, J. High Energy Phys. 08 (2017) 088.

[63] M. Cacciari and G. P. Salam, Phys. Lett. B 641, 57 (2006).

[64] M. Cacciari, G. P. Salam, and G. Soyez, J. High Energy Phys. 04 (2008) 063.

[65] M. Cacciari, G. P. Salam, and G. Soyez, Eur. Phys. J. C 72, 1896 (2012).

[66] J. Butterworth et al., J. Phys. G 43, 023001 (2016).

[67] S. Dulat, T.-J. Hou, J. Gao, M. Guzzi, J. Huston, P. Nadolsky, J. Pumplin, C. Schmidt, D. Stump, and C. P. Yuan, Phys. Rev. D 93, 033006 (2016).

[68] L. A. Harland-Lang, A. D. Martin, P. Motylinski, and R. S. Thorne, Eur. Phys. J. C 75, 204 (2015).

[69] R. D. Ball et al. (NNPDF Collaboration), J. High Energy Phys. 04 (2015) 040.

[70] A. Buckley, J. Ferrando, S. Lloyd, K. Nordstroem, B. Page, M. Ruefenacht, M. Schoenherr, and G. Watt, Eur. Phys. J. C 75, 132 (2015).

[71] N. Greiner, S. Höche, G. Luisoni, M. Schönherr, J.-C. Winter, and V. Yundin, J. High Energy Phys. 01 (2016) 169.

[72] F. Maltoni, E. Vryonidou, and M. Zaro, J. High Energy Phys. 11 (2014) 079.

[73] S. Forte and C. Muselli, J. High Energy Phys. 03 (2016) 122.

[74] F. Caola, S. Forte, S. Marzani, C. Muselli, and G. Vita, J. High Energy Phys. 08 (2016) 150. 